\title{
EL APORTE DEL DISEÑO INDUSTRIAL \\ EN EL CAMPO DE LA REHABILITACIÓN
}

\section{Mario Pinilla}

Universidad de los Andes

\section{Palabras claves}

Diseño Industrial, Terapia Ocupacional, Biomédica, Ingeniería Electrónica y Pedagogía.

\section{Introducción}

El desarrollo de los objetos en rehabilitación basado en el estudio de las relaciones de interacción que tienen con el ser humano, permite al Diseño In- dustrial enfatizar sus aspectos sociales, creativos y tecnológicos; así mismo, se contemplan los temas en un ámbito interdisciplinario.

\section{Objetivo general}

Presentar los aportes del Diseño Industrial generados en experiencias de investigación, procesos académicos y profesionales relacionados con la rehabilitación.

\section{Marco conceptual}

El estudio del hombre dentro de un marco de ocupación como aspecto crítico de la experiencia humana, busca comprender el sentido de una actividad a través de lo objetos y examina la interacción eficaz con un ambiente; este entorno compuesto por insumos generados de la cultura y la sociedad, es el que determina tareas que definen los objetos y sus formas de uso. 
Así mismo, con el estudio de las motivaciones, los hábitos, los roles, y gracias a la tendencia innata del ser humano a explorar como a dominar un ambiente [15] , se influye en la formación de destrezas que se evidencian en su desempeño y se permite definir aspectos de interacción, física, psicológica, perceptual, como enfoques que se relacionan con el Diseño.

\section{Metodología}

Para la propuesta se expondrán los siguientes temas:

- Experiencias académicas con instituciones en torno a la Terapia Ocupacional ${ }^{1}$.

- Igualmente se exponen dos ejemplos específicos de investigaciones realizadas por la Universidad de los Andes junto con instituciones:

\section{Proyecto 1: Tecnologías de apoyo para la comunicación Aumentativa y alternativa}

En esta investigación se desarrollan ayudas tecnológicas para fortalecer el uso de la Comunicación Aumentativa y Alternativa en procesos comunicativos de personas con necesidades especiales.

\footnotetext{
${ }^{1}$ Proyectos académicos desarrollados en le taller salud del Departamento de Diseño Industrial de la Universidad de los Andes, en colaboración con distintas instituciones de la salud en Bogotá.
}

Siempre que, se tengan en cuenta las necesidades reales del contexto, como base para proyectos de carácter científico-tecnológico y el desarrollo de artefactos cómodos en su uso para la población, por medio de la incorporación de tecnologías propias que reduzcan costos de producción y busquen el mejoramiento de la calidad de vida.
El proyecto se fundamenta en la importancia que adquiere para el ser humano con discapacidad la intervención pedagógica mediada por sistemas de comunicación distintos al habla; es frecuente que, debido a alteraciones en el desarrollo -bien por factores genéticos o adquiridos- estas personas presenten dificultades para comunicarse, lo cual implica una pérdida de deseo, así como de iniciativa para establecer interlocución con las demás, debido a la problemática misma y a la reacción de los otros.

Las dificultades en los procesos comunicativos han sido una constante en la atención de las personas con limitaciones y pueden generar o incrementar problemas de aprendizaje, por cuanto restringen el desarrollo de la lengua, del lenguaje, del pensamiento $y$, por supuesto, afectan el aprendizaje académico.

El objetivo se centra en, incorporar ayudas tecnológicas en los procesos de comunicación de personas con 
discapacidad que asisten a la Sala de Comunicación Aumentativa y Alternativa de la Universidad Pedagógica Nacional para observar la variación en la capacidad de interacción comunicativa

En cuanto a los resultados, Se implementaron once puestos de trabajó y su espacio adecuado para dicha sala, se fortalece la atención pedagógica que brinda, mediante tecnologías de apoyo para las personas con discapacidad que provienen de distintas instituciones y comunidades.

En esta investigación avalada por Colciencias, se desarrollan ayudas tecnológicas para fortalecer el uso de la Comunicación Aumentativa y Alternativa en procesos comunicativos de personas con necesidades especiales ${ }^{2}$.

\section{Proyecto 2: Rodilla pediátrica CIREC}

Este proyecto trata sobre el diseño de una nueva rodilla pediátrica protésica que permita generar una marcha segura para el paciente, además, esta debe ser adaptable a la infraestructura productiva Colombiana. La rodilla desarrollada en este trabajo es similar a otras existentes en el mercado, que poseen mecanismos de 4 barras, pero en la cara frontal no se implementa un componente que simula la rótula como parte de ella. Este vacío se complementa con la funda cosmética que usualmente es en espuma. El mecanismo diseñado tiene un rango amplio de flexión $\left(163^{\circ}\right)$, mientras que las con-

\footnotetext{
${ }^{2}$ Documento informe final entregado a colciencias, del proyecto "Tecnologías de apoyo para la comunicación aumentativa y alternativa".
}

vencionales alcanzan máximo $\left(145^{\circ}\right)$. En cuanto a otras investigaciones realizadas en el mundo, se ha trabajado en el diseño de rodillas geriátricas, y adultos en general con mecanismos hidráulicos de impulsión que las hace de elevado costo e inaccesibles para nuestra población, la rodilla diseñada en este trabajo cuesta aproximadamente $\$ 500.000$.

\section{Metodología empleada}

Se aborda el problema de diseño desde sus diferentes fases, etapas, actividades y técnicas, para diseñar el producto, tales como:

En el concepto estético-formal, se toma como analogía la rodilla fisiológica humana. Basado en el modelo del movimiento de la rodilla humana en el plano sagital [6], se menciona que una de las formas en que esta se mueve, es guiada por los ligamentos cruzados. Se realizaron maquetas y modelos escala natural (fig. 1) los cuales representan las características formales, con un acabado en blanco.

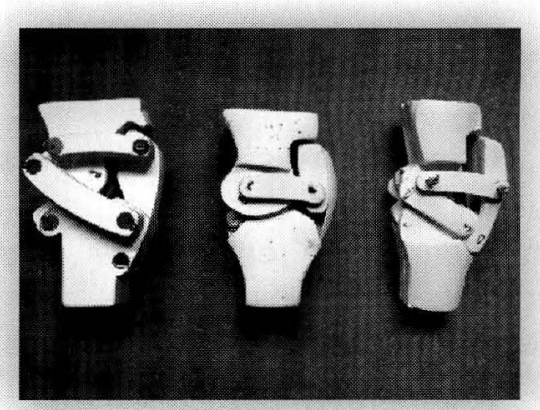

Figura 1. Primeras maquetas realizadas en cartón a escala 1:1.

En cuanto al diseño mecánico, se realizó interdisciplinariamente con un grupo 
de Ingenieros Mecánicos [16] (UNIANDES), se logra las mejoras de la primera geometría de rodilla ya ideada. El proceso inició con la evaluación cinemática de la última alternativa en maqueta (fig.1), para lo cual se realizó la digitación del mecanismo con el software SOLID EDGE en 3D. Se implementó el método de análisis de elementos finitos bajo el criterio de falla de Von Mises [16], que permite observar el comportamiento estructural del material ante una carga de *2000 New en forma estática.

Se realizaron pruebas para conocer el desempeño de la rodilla pediátrica en usuarios transfemorales, en el ciclo de la marcha protésica. Los criterios de selección de los pacientes como una población homogénea, deben cumplir con características como; experiencia en el uso de prótesis mayor a 6 meses, muñón transfemoral (estándar, largo), se excluyen pacientes bilaterales, que utilicen ayudas técnicas como caminador, muletas, silla de ruedas o con enfermedades sistémicas asociadas.

Resultados: Esta se construyó y se probó hasta la fecha en 5 pacientes, la cual presentó un buen desempeño en la adaptación, funcionalidad, fabricación y resistencia. Se realizó su validación en diferentes ámbitos; rehabilitación, biomecánica de la marcha, opinión de satisfacción por parte de los usuarios y adaptación de la misma a la fabricación de la prótesis, lo cual demostró su utilidad. En la biomecánica, no genera ningún inconveniente en el ciclo de marcha de los usuarios y ofreció ventajas en tiempo de apoyo como mejor movilidad de la cadera. Con respecto a los usuarios, les permitió el desempeño en actividades cotidianas como caminar en terrenos irregulares, inclinados, realizar actividades lúdicas entre otras. En la fabricación, presentó una fácil adaptación con los componentes modulares existentes en CIREC (alienador, adaptador al socket, adaptación al tubo de pantorrilla, tobillo y pie), sin embargo, los problemas principales detectados en la evaluación, se relacionan con la dificultad para adaptar muñones largos de amputados transfemorales, además, en la fabricación de las prótesis, se deben explorar alternativas a cerca de la terminación cosmética.

\section{Conclusiones y recomendaciones}

A medida que se realizan estas experiencias interdisciplinarias, el conocimiento y la experticia de los profesionales de este sector de la salud y la tecnología, son aprovechadas por el Diseño Industrial para aportar en un proceso innovador tecnológico que realiza las transformaciones necesarias para potenciar las capacidades residuales de las personas con alguna discapacidad.

NOTA: Estas experiencias se complementarán con una muestra de afiches que ilustran el trabajo con instituciones en torno a la Terapia Ocupacional y la investigación en Salud. 


\section{Bibliografía}

1. Henao Luis Fernando. 1993. Las prótesis en polipropileno una alternativa más. Fundación CIREC Bogotá.

2. Fitzsimons Steve. 2001, Prótesis pediátricas: sus demandas singulares, O\&P world, Vol 4., No.2.

3. JPO Journal of prosthetics and orthotics 1992 Cuidados para niños con necesidades ortopédicas Vol 4 jul Amputaciones y prótesis London England Ed. Jims

4. Breakey James.1998 Mas allá de la rodilla de cuatro barras, JPO Vol 3, pg. 77-80.

5. Kapandji A.I. Fisiología articular 2 Miembro inferior. Ed. Medica Panamericana-Malione. Madrid España. 1998.

6. Ramiro José. Guía de recomendaciones para el diseño de calzado IBV 1995.

7. http://www.shotokanryukaseha.com/otrostextos/aparato_locomotor.htm

8. J.K.Chakraborty, K.M. Patil, India. "A new modular six-bar linkaje trans-femoral prosthesis for walking and squatting". ISPO. Agust, 1994, vol 18, No 2.

9. R. Pawlik "Gait analytical assessment of the joint adjustment and the prosthetic aligment by using the interior C-Leg sensor system"C/ o Otto Bock Austria- Kaiserstr. 2002.

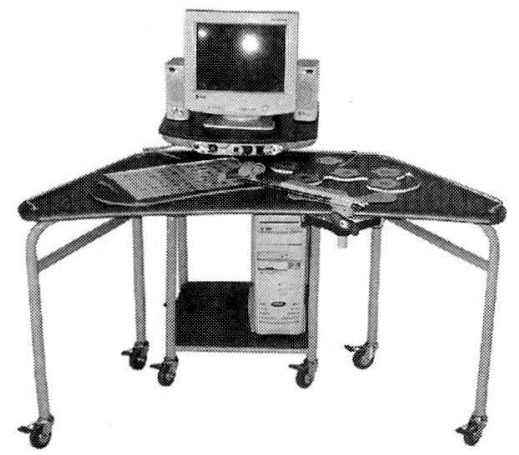

10. Información técnica sobre tipologías de rodilla de 4 ejes como, Technical Report on the TGK-5PSOSLK, TGK-5PSO and the TGK-50SO PC Knee Units, Relased from DAW industries Department of Research and Development. 2003.

11. Mohan D., Sethi P.K., India, Mathematical modelling end field trials of ain inexpesive endoskeletal above-knee Prosthesis. ISPO, Vo.6,No.2, 1992.

12. Donker Stella \& Beek Peter, Interlimb coordination in prosthetic walking: Effects of asymmetry and walking velocity. Acta Physiológica, 2002.

13. Hill-Watson Selena, Report on the evaluation of the DAV/Seattle Knee, Journal of rehabilitation Research and Development, Vol 31, No.1, 1994.

14. Ellepola Wijegupta, Sheredos Saleem J, Report on the Evaluation of the VA/SEATTIE Below-knee Prótesis, Journal of Rehabilitation Research and Development, 1993.

15. Terapia ocupacional, H. Hopkins/ H. Smith. ED. Medica panamericana, p. 76.

16. Moncaleano John, Proyecto de grado: Desarrollo y evaluación de prótesis para niños con amputaciones transfemorales. Dpto. de Ingeniería Mecánica. UNIANDES, 2003.

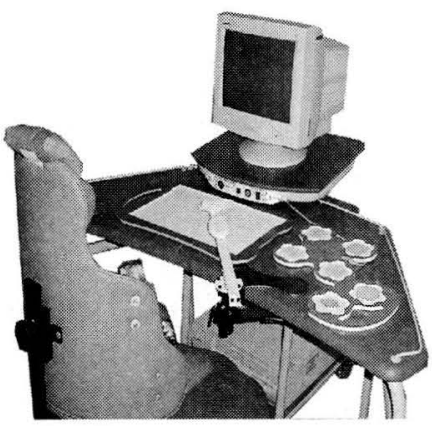

